\title{
Multi-modal human-machine control interfaces of upper limb motorized exoskeletons for severely impaired patients*
}

\author{
S. Dalla Gasperina ${ }^{\S}$, Student Member, IEEE, M. Gandolla ${ }^{\S}$, A. Calcagno, A. Costa, L. Aquilante, \\ M. Puchinger, M. Gfoehler, F. Braghin, A. Pedrocchi, Member, IEEE
}

\begin{abstract}
The aim of this study concerns the evaluation and comparison of different Human-Machine Interfaces for the control of an upper limb motorized exoskeleton for severely impaired patients. Different approaches (i.e. manual, vocal, visual control) are tested in a simulation environment on three subjects affected by muscular dystrophy with the aim of assessing the capability of the system to interact with the user and vice versa. A Graphical User Interface shows the simulated behavior of the exoskeleton to the user which has to perform reaching tasks in the space by moving the exoskeleton endeffector to defined virtual targets that are displayed on the screen. Specific assessment of the interaction of the user with each control interface is achieved, while a quantitative evaluation of the usability of all the three approaches is provided by a System Usability Scale (SUS) questionnaire. All patients were able to interact with all control interfaces without difficulties and to complete reaching tasks in simulation. SUS scores showed overall good usability of the Human-Machine Control Interfaces suggesting that the manual and the vocal control interfaces are preferred by the subjects.
\end{abstract}

\section{INTRODUCTION}

Neuromuscular diseases are pathologies that gradually affect the muscular functionality of the entire body. Specifically, muscular dystrophy (MD) is a disorder that presents no central or peripheral nervous system involvement, but it is characterized by the progressive deterioration of the muscular membrane [1]. This pathology firstly affects lower limbs, leading to the loss of ambulation capabilities, and, as the disease advances, also the control of upper limbs is increasingly compromised [2], [3]. Nowadays there are no available cures or rehabilitation solutions that allow people affected by muscular dystrophy to restore muscular functionalities. The pathology progression leads to an increasing difficulty in the execution of daily life activities (DLA) without assistance and, consequently, to a progressive decrease of patients' autonomy. In particular, the decreased control of upper extremities causes a strong dependence on the caregivers and psychological stress for the patient [4].

*Research supported by BRIDGE and EMPATIA projects funded by Fondazione Cariplo in collaboration with Regione Lombardia.

$\S$ S.D.G and M.G. have equally contributed to this work and are the corresponding authors

S. Dalla Gasperina, A. Calcagno, A. Costa, L. Aquilante, F. Braghin and A. Pedrocchi are with Politecnico di Milano, Milano, Italy (email: stefano.dallagasperina@polimi.it; $\quad$ alessandra.calcagno@mail.polimi.it; andrea.costa@polimi.it; $\quad \underline{\text { lorenzo.aquilante@polimi.it; }}$ francesco.braghin@polimi.it; alessandra.pedrocchi@polimi.it )

M. Gandolla is with Politecnico di Milano, Polo territoriale di Lecco, Lecco, Italy (e-mail: marta.gandolla@polimi.it ).

M. Puchinger and M. Gfoehler are with the Institute of Engineering Design and Logistics Engineering, TU Wien, Vienna, Austria (e-mail: markus.puchinger@tuwien.ac.at; margit.gfoehler@tuwien.ac.at ).
In this regard, several upper limb robotic orthoses are currently available on the market, but none of them seems to adapt to the specific needs of muscular dystrophy patients at most severe stages, when the loss of upper limbs function is almost complete [5]. For MD patients affected by total upper limbs paralysis, a powered exoskeleton able to actively move multiple joints might be the solution [6].

Among the commercial available devices [7], it is possible to find bulky exoskeletons, mainly used for rehabilitation purposes (e.g. MIT-MANUS developed by the Massachusetts Institute of Technology, USA [8]), passive exoskeletons (e.g. WREX by JAECO Orthopedics, USA [9]) and active exoskeletons (e.g. Ayura by Armon Products, Netherlands [10]) that can be easily mounted on the wheelchair but which require the user to preserve some residual muscular activity, and external manipulators (e.g. JACO robotic arm by Kinova Robotics, Canada [11]). The latter solution seems to be the only one able to adapt to all the stages of muscular dystrophies. Nevertheless, this kind of devices completely replaces the arm function, depriving the patient of the execution of the desired task with his/her own body. Indeed, from a psychological point of view, performing a task directly with a continuous control of the movement is a huge achievement for these patients, even if passively moved by an external device [12], [13].

In this scenario, the BRIDGE project is based on a powered exoskeleton able to reduce the physical and psychological impairment of patients affected by neuromuscular diseases, such as Muscular Dystrophy. The BRIDGE system consists of a wearable upper limb motorized exoskeleton and a PC-based control system aimed at interpreting the patient's movement intentions and translating them into real movements of the exoskeleton.

The BRIDGE exoskeleton (patent pending) [14] is a lightweight motorized mobile arm exoskeleton with gravity support which consists of four modules that are connected with carbon rods and tubes and are fixed by using clamping mechanisms which additionally enable to adjust the exoskeleton to various arm sizes. Five Degrees of Freedom (DOFs) allow the user to explore the workspace in terms of position and orientation of the end-effector (i.e., hand position). Three DOFs are provided at shoulder level: 1) shoulder abduction/adduction in the horizontal elevation plane; 2) shoulder flexion/extension in the sagittal plane; 3 ) shoulder internal/external rotation along the humerus' axis; one DOF is set at elbow level (elbow flexion/extension) and one DOF involves wrist pronation/supination (Fig. 1). Stepper motors with harmonic drive gearboxes paired with optical encoders actively control the motion of each degree of freedom of the exoskeleton. 


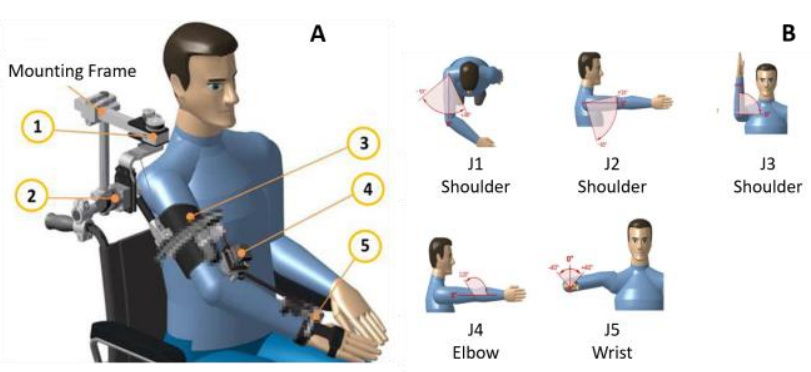

Figure 1. A) Representation of the BRIDGE powered exoskeleton; B) BRIDGE Degrees Of Freedom (DOFs)

As it concerns the control strategy, it can be divided in three main steps:

1) Derivation of the current position and orientation of the end-effector - On the basis of the forward kinematic model of the exoskeleton (defined according to the DenavitHartenberg convention [15]) given the angular position of the joints, the three-dimensional Cartesian position of the end-effector is computed. The orientation of the endeffector, instead, is directly determined by the $5^{\text {th }}$ DOF (wrist pronation /supination).

2) Definition of the desired end-effector position Starting from the user's inputs, the desired end-effector position can be computed thanks to the combination of its known actual position and the desired displacement. An algorithm assesses whether the desired position of the endeffector belongs to the workspace of the exoskeleton, whose external boundaries are defined taking into account the patient-specific ranges of motion.

3) Definition of the desired joints angular position - The target angular positions to be used for motors control are computed by means of an inverse-kinematic algorithm [16]. Since four DOFs are used to determine the threedimensional Cartesian position, the derived kinematic redundancy, which is also present in the physiological human arm, assures a reliable computation of the inversekinematic avoiding singularities and minimizing joint torques required to compensate gravity. The inversekinematic algorithm performs an optimization process which takes into account not only the actual and the desired position, but also boundary conditions for all DOFs and distance from the nearest robotic singularity.

While the design, modeling and development of the exoskeleton and of its control strategy have been discussed previously [5], the present work is focused on the comparison and evaluation of different multi-modal interfaces for the control of the exoskeleton. Indeed, different interfaces have been investigated in collaboration with the UILDM (Unione Italiana Lotta contro la Distrofia Muscolare - Italian National Association Fighting Muscular Dystrophy) in order to adapt the interaction between the robotic arm and the user in compliance with the target pathology, with different levels of disability and, in the case of neurodegenerative diseases, with the progress of the impairment. In particular, since different approaches may be preferred according to the context of use and to the residual capability of the patient, in this study the subject has the possibility to control the position and the orientation of his/her own upper limb end-effector by freely choosing among three different approaches: manual control, vocal control and visual control.

Since patients affected by neuromuscular disorders are already used to moving their motorized wheelchairs with a joystick-based control system, the manual control interface that precisely exploits the use of a joystick has been firstly considered as the most promising strategy [17]. As a matter of fact, patients own an improved tactile sensibility in the hands that allows them to perform fine movements, and, at the same time, they already master the cognitive process by which a small displacement of the joystick corresponds to a larger movement in a different space (i.e. wheelchair movements).

From another point of view, speech recognition is nowadays gaining increased interest and the related technological development is making it accessible to most common uses. Several studies have been conducted in the context of patients affected by motor disorders and humanmachine interfaces based on speech recognition have already been developed [18], [19]. Indeed, such an approach may allow subjects, who lost the hand/arm functionality, to interact with the environment and to perform daily living activities by controlling and mastering the motion of an exoskeleton or of an external manipulator [20]. Nevertheless, voice-recognition control interfaces are dependent on the background noise and on the impairment of patients that in the most compromised cases may present communicative and respiratory issues.

Both previous approaches share the hypothesis that patients still own residual functionalities in terms of hand fine movements or speech capabilities. Like most neuromuscular disorders, muscular dystrophy is degenerative, and therefore an advanced stage of the disease may lead the patient to preserve his/her cognitive abilities and, at the same time, to progressively lose the use of speech and limbs mobility. For these reasons, a visual control interface has been introduced as an alternative solution for the most impaired patients, who are not suitable for the other approaches.

\section{HUMAN-MACHINE CONTROL INTERFACES (HMCIS)}

According to the specific needs of the patients, three different approaches have been considered. The user, by means of manual control, vocal control or visual control should be able to directly control the position and orientation of the end-effector of the exoskeleton (i.e., hand) by providing the intention to move in a desired direction from the actual position, and to change wrist pronation/supination. Each of these Human-Machine Control Interfaces (HMCIs) receives inputs from the patient, which are representative of the will to move in a particular direction. The directions of interest that can be controlled by the user are $\mathrm{x}, \mathrm{y}, \mathrm{z}$ displacements of the end-effector and the $\theta_{5}$ wrist pronation/supination angle. Moreover, ad-hoc procedures have been implemented for each HMCI to enable/disable the control mode and to enter an "Emergency Mode" which stops the motion of the exoskeleton and activates an acoustic alarm. 


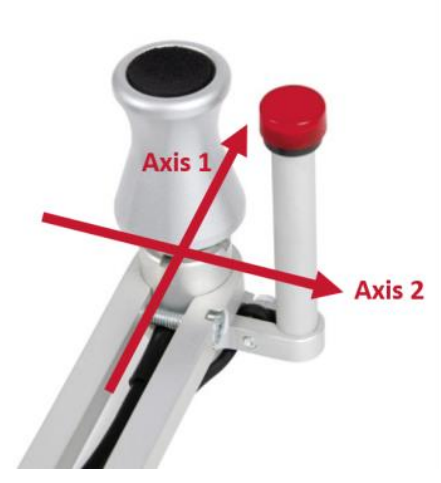

Figure 2. Ottobock 2-axis high-sensitivity Mini-Joystick with integrated momentary push button

\section{A. Manual Control Interface (MCI)}

The selected input hardware is a 2-axis high-sensitivity Mini-Joystick (Ottobock, Germany) (Fig. 2), a momentary push button, and a dedicated Human Interface Device (HID) acquisition board that processes the analog data from the joystick. Since the joystick is based on a 2-axis system while the user's intentions can be along four directions (x, y, z, $\theta_{5}$ ), there is the possibility (through a dedicated button) of switching the control mode to move the end-effector in the $\mathrm{x}$ y horizontal plane rather than along $\mathrm{z}, \theta_{5}$ directions. Given its high sensitivity, the joystick requires a calibration procedure that normalizes the input values with respect to the maximum patient-specific displacement, along the two directions for each axis.

\section{B. Vocal Control Interface (VoCI)}

In this study, a voice-independent human-machine control interface has been developed based on the Google Cloud Speech-to-text API [21]. The vocal control interface is implemented within a PC-based control system that continuously listens and detects words and sentences from the user. An external microphone is used in order to minimize disturbances coming from the environment. After the activation of the control with a specific keyword, the user is able to control the motion of the end-effector of the exoskeleton by means of standard instructions. The associated displacement is a patient-dependent parameter, and might be small, medium or large according to the patient's selection via vocal-control. In agreement with the UILDM, a dictionary of Italian key-words has been defined as a set of commands to be used by the user to indicate the different directions of motion.

\section{Visual Control Interface (ViCI)}

The visual control interface relies on an advanced version of the traditional Pupil Centre Corneal Reflection (PCCR)[22] fixation detection technology that allows to interpret the intentions of the user by tracking the gaze position and taking into account any head movements. A table mounted eye-tracker (Tobii T60 manufactured by Tobii, Sweden) positioned at about $70 \mathrm{~cm}$ from the user is able to compute the estimation of the position of the eye in space and the fixation point with an intrinsic accuracy of 0.5 degrees. Considering a 24" screen with a resolution of $1280 \times 1024$ pixels, the accuracy of the eye-tracker to detect the point of gaze, in the assumption of a perfect calibration, corresponds to about 2 pixels at the screen level. A dedicated Graphical User Interface guides the patient through the procedure: the user is asked to fix for an adjustable amount of time different areas of the screen (identified by virtual buttons) according to the action to be performed (i.e. set the direction of the movement or the orientation of the end-effector, enable/disable the control mode or activate safety mode). Since the user has to look at the screen to set commands during the execution, he/she cannot see the actual motion of the exoskeleton, therefore a video streaming showing the working volume of the exoskeleton is displayed at the center of the screen and the user can ask to adjust the field of view according to his/her needs.

\section{MethodS}

The experimental protocol of this study provides a first assessment of the interaction between the user and each human-machine control interface. Then, the subject is asked to perform reaching tasks in a simulation environment. Finally, a quantitative evaluation of the usability of all approaches is provided by a System Usability Scale (SUS) questionnaire [23]. Participants of this study are three voluntary subjects affected by different types of muscular dystrophy (Table I). Patients are characterized by various levels of impairment that were assessed by the Performance of Upper Limb (PUL) score [24], according to which higher values correspond to less impairment of the subject. Subject 3 , the most compromised, could not be evaluated on the PUL scale since his residual functionalities were below the minimum level. The objective of the study and the experimental protocol have been exposed to the patients and their caregivers, and participants were asked to sign a written informed consent.

\section{A. Evaluation of the human-machine interaction}

1) Manual Control Interface (MCI) - after the calibration procedure to normalize the joystick output according to the maximum residual capabilities of the patient, in order to quantitatively assess the capability of the system to follow user's intentions, the displacement of the joystick is compared with the actual movement of the simulated exoskeleton. For each three-dimensional axis, the correlation coefficient is computed between the normalized joystick signal and the normalized difference between desired and current positions.

2) Vocal Control Interface (VoCI) - the verification of the correct recognition of the vocal commands is performed by asking the user to pronounce seven times each word of the dictionary. Accuracy is computed as the ratio between the number of correct detections against the overall number of attempts.

3) Visual Control Interface (ViCI) - after a standard eyetracking calibration procedure ( 30 seconds), the user is asked to steadily fix on the screen a target square of 50 pixels, and the accuracy of the fixation detection system is achieved by computing the error in pixels between the center of the target and the actual fixation point identified by the patient. The procedure is repeated for each patient with three different visual targets. 
TABLE I. DEMOGRAPHIC CHARACTERISTICS OF THE PARTICIPANTS

\begin{tabular}{|c|c|c|c|c|}
\hline Subject & Age & Sex & Type of Dystrophy & PUL score $^{\text {a }}$ \\
\hline Subject 1 & $18 y$ & Female & Limb-Girdle (LGMD) & 23 \\
\hline Subject 2 & $23 y$ & Male & Limb-Girdle (LGMD) & 24 \\
\hline Subject 3 & $32 \mathrm{y}$ & Male & Duchenne (DMD) & N.A. \\
\hline
\end{tabular}

On the basis of these results the virtual buttons for the control of the exoskeleton are sized in order to avoid misdetection of the commands.

\section{B. Reaching tasks in simulation environment}

After the evaluation of the capability of the system to interpret and translate the user's intentions, the user is asked to reach virtual targets by controlling the end-effector of the exoskeleton. The aim of these tests is to assess if the patients are able to interact with each of the HMCIs to perform reaching tasks in a simulation environment. A dedicated GUI has been developed to show on screen the simulated avatar of the exoskeleton, which is used by the user as a visual feedback (Fig. 3). The VoCI approach involves the activation (through "Jarvis" keyword) and the subsequent interaction with the vocal human-interface, which guides the patient by means of vocal instructions and vocal feedbacks. For what concerns the ViCI, the fixation time plays a crucial role for the selection of the correct command: the user has to fix the target buttons on the screen for a certain amount of time to correctly activate the command in order to avoid unwanted detection and to remove artifacts related to head movements. Each exercise is repeated three times for each patient, at each repetition the virtual target to be reached is set to a different $3 \mathrm{D}$ point.

\section{Human-Machine Control Interfaces usability}

The assessment of the usability of the system has been carried out by means of the SUS questionnaire that was filled in by the patients at the end of the trials. The SUS survey investigates the perceived overall ease of use of the system as the combination of effectiveness, efficacy and usefulness of the system. SUS scores are computed according to Brooke's guidelines [25]. Moreover, additional questions were asked to the subject with the aim of assessing their propensity towards one of the proposed HMCIs. In particular, patients were asked to choose the preferred interface and the easiest interface of use, and to indicate which control approach they would prefer to be implemented in a real environment.

\section{RESULTS}

\section{A. Evaluation of human-machine interaction}

1) Manual Control Interface (MCI) - all patients were able to correctly interact with the joystick-based control interface. As expected, the correlation, computed as the Pearson bivariate correlation coefficient, suggests a perfect relationship between the displacement of the joystick and the effective movement of the end-effector (i.e. all mean values $>0.99$ ). In fact, during the execution of the trials, the visual feedback assured the correct behavior of the control system, i.e. the joystick displacement in a certain direction corresponded to a movement of the virtual end-effector in the same direction. An example of the acquired signals during a test is shown in Fig. 4, which represents the differential position and the displacement of the joystick for each cartesian axis.

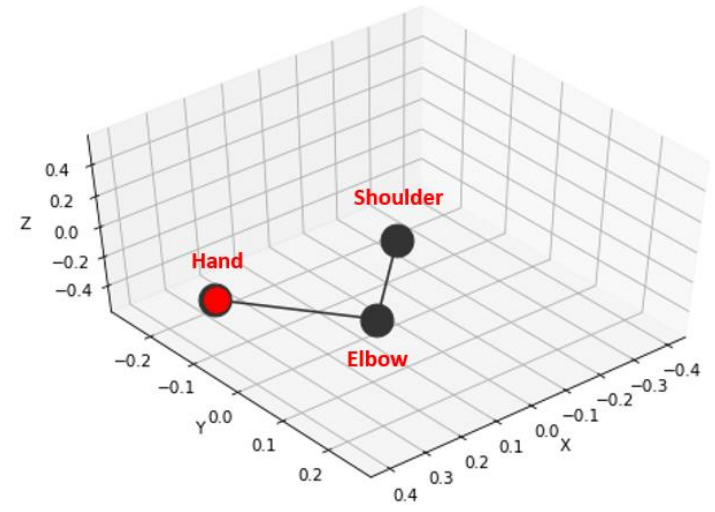

Figure 3. View of the simulation environment
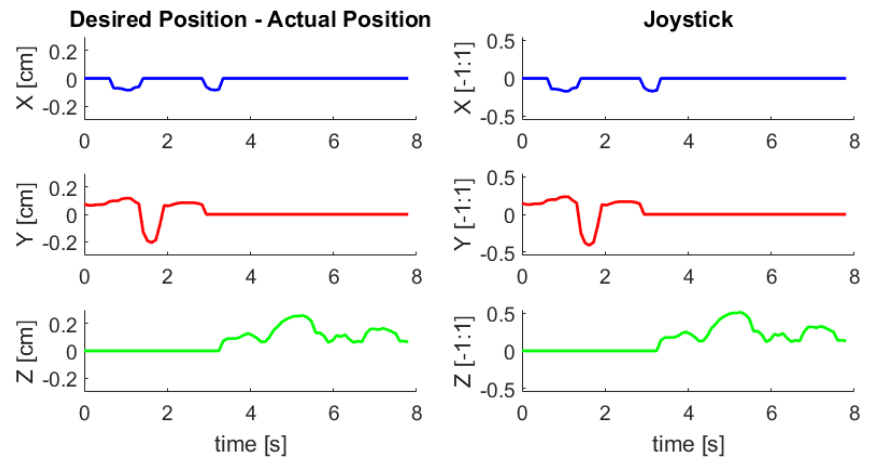

Figure 4. Left Panel: difference in centimeters between desired position and actual position along $\mathrm{x}, \mathrm{y}$ and $\mathrm{z}$ axis; Right Panel:

joystick input normalized between -1 and +1 along $\mathrm{x}, \mathrm{y}$ and $\mathrm{z}$ axis.

TABLE II. VOCAL RECOGNITION ACCURACY

\begin{tabular}{|c|c|c|c|c|}
\hline \multirow{2}{*}{ Command } & \multirow{2}{*}{ Function } & \multicolumn{3}{|c|}{ Accuracy [\%] } \\
\cline { 3 - 5 } & & Subject 1 & Subject 2 & Subject 3 \\
\hline "Jarvis" & Enable & 100.0 & 100.0 & 100.0 \\
\hline "Fermo" & Stop & 85.7 & 100.0 & 100.0 \\
\hline "Avanti" & Forward & 100.0 & 100.0 & 100.0 \\
\hline "Indietro" & Backward & 100.0 & 100.0 & 71.4 \\
\hline "Destra" & Right & 85.7 & 100.0 & 85.7 \\
\hline "Sinistra" & Left & 85.7 & 100.0 & 100.0 \\
\hline "Salire" & Up & 85.7 & 100.0 & 100.0 \\
\hline "Scendere" & Down & 100.0 & 100.0 & 100.0 \\
\hline "Riposo" & Sleep & 71.4 & 100.0 & 100.0 \\
\hline "Dormi" & Sleep & 100.0 & 100.0 & 85.7 \\
\hline "Termina" & Quit/Exit & 100.0 & 85.7 & 100.0 \\
\hline Overall ${ }^{a}$ & $100.0(14.3)$ & $100.0(0.0)$ & $100.0(7.1)$ \\
\hline
\end{tabular}


2) Vocal Control Interface (VoCI) - concerning the validation of the recognition of vocal commands, results are shown in Table II. For this study, values of accuracy higher than $70 \%$ are considered acceptable, although the recommended threshold should be around $90 \%$. The median values show good accuracy of commands' detection, independently from the level of impairment of the patients.

3) Visual Control Interface (ViCI) - three points per patient were detected, the accuracy of the fixation detection technology has been evaluated by computing the differences in pixels between the center of the target points and the actual fixation points detected by the eye-tracker. Calibration procedure resulted to highly influence the accuracy of fixation measurements, however all subjects were able to interact with the ViCI with sufficient precision. Results show errors up to about 30 pixels (Table III). Such distances of misdetection were considered acceptable with respect to the dimensions of the screen, being in the worst case equal to about $3 \%$ of the smaller size of the screen (i.e. 1024 pixels). This observation leaded to the development of a GUI with virtual buttons of about 200 pixels (i.e. much wider than the maximum fixation error) that should permit an accurate control detection.

\section{B. Reaching tasks in simulation environment}

An exemplificative trial showing the three-dimensional trajectory of the end-effector during a reaching task is shown in Fig. 5. All patients completed the exercises successfully. Indeed, all subjects were able to move the exoskeleton in the desired directions by handling the joystick and pushing the button to complete the exercises by reaching the virtual target.

Moreover, they were able to activate the vocal assistant by pronouncing the "Jarvis" command, to move the end-effector to the target points by means of movement vocal commands, to stop the movement (Stop command), to stand-by the vocal assistant (Sleep command) and to quit the control software. The visual control was suitable for the control of the exoskeleton as well and the subjects encountered no issues in reaching the virtual targets.

\section{Human-Machine Control Interfaces usability}

Detailed answers provided by the patients in evaluating the usability of the different human-machine control interfaces are reported in Table IV. In particular, two out of three subjects preferred the VoCI, while one subject selected the manual interface as the most usable and easiest of use.

TABLE III. VISUAL FIXATION DETECTION ERRORS

\begin{tabular}{|c|c|c|c|c|c|c|}
\hline \multirow{2}{*}{$\begin{array}{c}\text { Target } \\
\text { point }^{\mathbf{a}}\end{array}$} & \multicolumn{3}{|c|}{ Detected point $^{\mathbf{a}}$} & \multicolumn{3}{c|}{ Error [pixel] } \\
\cline { 2 - 7 } & Subj.1 & Subj.2 & Subj.3 & Subj.1 & Subj.2 & Subj.3 \\
\hline$X=525$ & 506.56 & 504.54 & 523.30 & 21.42 & 20.48 & 9.72 \\
$Y=525$ & 535.90 & 524.23 & 534.57 & & & \\
\hline$X=615$ & 616.64 & 586.83 & 647.33 & 14.61 & 28.35 & 33.35 \\
$Y=615$ & 629.52 & 618.20 & 623.19 & & & 22.71 \\
\hline$X=275$ & 274.00 & 251.69 & 266.47 & 8.35 & 34.97 & 22.05 \\
$Y=275$ & 283.29 & 301.07 & 296.05 & \multicolumn{5}{|c|}{} \\
\hline \multicolumn{7}{|c|}{${ }^{2}$ target and detected points are expressed in terms of screen x, y coordinates in pixels } \\
\hline
\end{tabular}

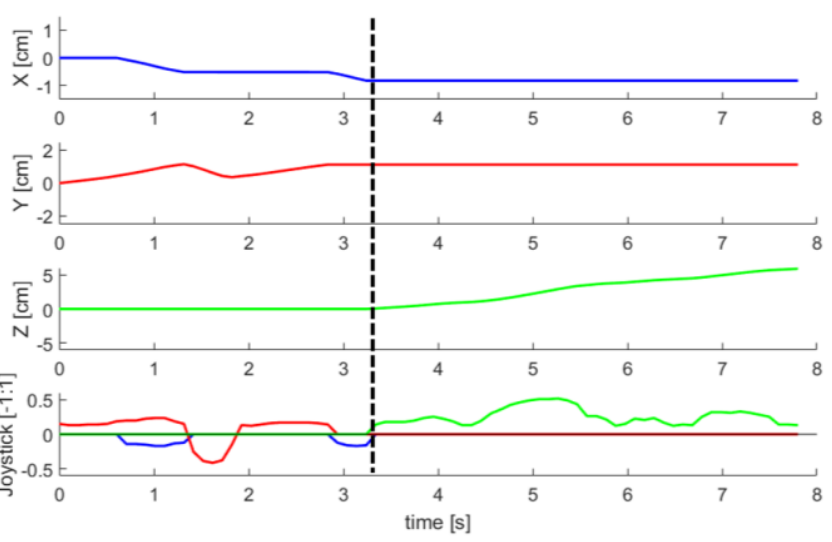

Figure 5. End-Effector trajectory along $\mathrm{x}, \mathrm{y}$ and $\mathrm{z}$ axis (first 3 plots), and joystick input signals (last plot). Vertical dotted line indicates the time instant when the push button is pressed.

TABLE IV. HMCIS USABILITY RESULTS

\begin{tabular}{|c|c|c|c|}
\hline & Subject 1 & Subject 2 & Subject 3 \\
\hline SUS - Joystick ${ }^{\mathrm{a}}$ & 100.0 & 82.5 & 95.0 \\
\hline SUS - Vocal ${ }^{\mathrm{a}}$ & 82.5 & 90.0 & 100.0 \\
\hline SUS - Gaze ${ }^{a}$ & 90.0 & 82.5 & 90.0 \\
\hline Preferred HMCI & Joystick & Vocal & Vocal \\
\hline Easiest HMCI & Joystick & Vocal & Vocal \\
\hline
\end{tabular}

\section{DISCUSSION AND CONCLUSIONS}

Considerable effort has been recently devoted to the improvement of the quality of life of people affected by neuromuscular disorders during daily life activities. Most of the issues related to the reduced mobility and respiratory failure, essential to survival, have been overcome thanks to the advent of recent technologies. However, the loss of functionalities of the upper limbs causes a strong dependence on caregivers and a psychological stress for the patient. The BRIDGE project has been designed with the aim of answering to the need of dedicated devices for the assistance and/or support of arm functions, by developing a motorized exoskeleton adaptable to different stages and types of muscular dystrophy. According to the specific needs of the patients, three different approaches have been considered. The user should be able to directly control the position and orientation of the end-effector of the exoskeleton (i.e. hand), by means of manual control, vocal control or visual control. Specifically, this work concerns the evaluation of the capability of the systems to interact with the user and vice versa. Such interfaces have been tested, evaluated and compared in a simulation environment on three voluntary dystrophic patients.

Concerning the Manual Control Interface, the high sensitivity of the joystick - key feature of this approach allowed the detection of the smallest displacement performed by the most impaired subjects. At the same time, in order to be adaptable to different levels of disability, the joystick required a calibration procedure for the adaptation of the control interface to the patients' needs. As expected, the effective movements of the virtual end-effector correctly 
reflected the subjects' intentions in the simulation environment. Further validation will be performed in a real environment, where inertia and dynamics of the system will be considered.

About the Vocal Control Interface, independently on the level of impairment, all patients were able to interface the vocal control interface, and to apply with high accuracy their intentions of movement by means of vocal instructions. The most compromised subject (i.e., subject 3), required occasional non-invasive ventilation during the trials to prevent respiratory failure. Nevertheless, the use of the mechanical ventilator during the execution of the tests did not significantly influence the capability of the subject to interact with the control system.

Fixation detection errors were adequate to exploit the Visual Control Interface to command the virtual exoskeleton. However, such fixation errors suggest that a more accurate technology for eye-tracking could be examined for a direct control of the end-effector: the target three-dimensional position should be identified by combining gaze position for the vertical and horizontal axes, and the eye vergence for the depth, thus avoiding virtual buttons.

All patients were able to interact with all control interfaces without difficulties and to complete reaching tasks in simulation. Although the number of patient was limited, the usability has been positively evaluated [26], [27] for all the HMCIs, suggesting an overall good satisfaction of the users when interfacing with the control system of the exoskeleton. Overall, the MCI and VoCI approaches were preferred by the subjects since the visual HMCI constrained them to fix the eye-tracking screen, preventing them to directly look and monitor the behavior of the exoskeleton. However, since no statistical evaluation of the results was possible, further studies should be conducted on a more numerous population of subjects to fully evaluate the presented Human-Machine Interfaces.

\section{REFERENCES}

[1] A. E. H. Emery, "The muscular dystrophies," in Lancet, 2002, vol. 359 , no. 9307 , pp. $687-695$.

[2] J. L. Herder, N. Vrijlandt, T. Antonides, M. Cloosterman, and P. L. Mastenbroek, "Principle and design of a mobile arm support for people with muscular weakness," J. Rehabil. Res. Dev., vol. 43 , no. 5, p. 591, 2006.

[3] A. G. Dunning and J. L. Herder, "A review of assistive devices for arm balancing," in IEEE International Conference on Rehabilitation Robotics, 2013, vol. 2013, p. 6650485.

[4] M. A. Grootenhuis, J. de Boone, and A. J. van der Kooi, "Living with muscular dystrophy: Health related quality of life consequences for children and adults," Health Qual. Life Outcomes, vol. 5, no. 1, p. 31, Jun. 2007.

[5] M. Gandolla et al., "BRIDGE - Behavioural reaching interfaces during daily antigravity activities through upper limb exoskeleton: Preliminary results.," IEEE Int. Conf. Rehabil. Robot., vol. 2017, pp. 1007-1012, Jul. 2017.

[6] G. R. Johnson, D. A. Carus, G. Parrini, S. S. Marchese, and R. Valeggi, "The design of a five-degree-of-freedom powered orthosis for the upper limb," Proc. Inst. Mech. Eng. Part H J. Eng. Med., vol. 215, no. 3, pp. 275-284, Mar. 2015.
[7] L. A. Van Der Heide, B. Van Ninhuijs, A. Bergsma, G. J. Gelderblom, D. J. Van Der Pijl, and L. P. De Witte, “An overview and categorization of dynamic arm supports for people with decreased arm function," Prosthet. Orthot. Int., vol. 38, no. 4, pp. 287-302, Aug. 2014.

[8] B. J. F. Driessen, H. G. Evers, and J. A. V. Woerden, "MANUS A wheelchair-mounted rehabilitation robot," Proc. Inst. Mech. Eng. Part H J. Eng. Med., vol. 215, no. 3, pp. 285-290, Mar. 2015.

[9] T. Estilow et al., "The Wilmington Robotic Exoskeleton (WREX) improves upper extremity function in patients with Duchenne muscular dystrophy," Neuromuscul. Disord., vol. 24, no. 9-10, pp. 824-825, Jan. 2014.

[10] B. Mastenbroek, E. De Haan, M. Van Den Berg, and J. L. Herder, "Development of a mobile arm support (Armon): Design evolution and preliminary user experience," 2007 IEEE 10th Int. Conf. Rehabil. Robot. ICORR'07, vol. 00, no. c, pp. 1114-1120, 2007.

[11] V. Maheu, P. S. Archambault, J. Frappier, and F. Routhier, "Evaluation of the JACO robotic arm: Clinico-economic study for powered wheelchair users with upper-extremity disabilities," in IEEE International Conference on Rehabilitation Robotics, 2011, pp. $1-5$.

[12] M. Gandolla et al., "Artificial neural network EMG classifier for functional hand grasp movements prediction.," J. Int. Med. Res., vol. 45, no. 6, pp. 1831-1847, Dec. 2017.

[13] G. Tacchino, M. Gandolla, S. Coelli, R. Barbieri, A. Pedrocchi, and A. M. Bianchi, "EEG Analysis During Active and Assisted Repetitive Movements: Evidence for Differences in Neural Engagement," IEEE Trans. Neural Syst. Rehabil. Eng., vol. 25, no. 6, pp. 761-771, Jun. 2017.

[14] M. Puchinger, "Development of a mobile motorized arm exoskeleton." MSc thesis, TU Wien, 2015.

[15] Denavit and R. Hartenberg, "A kinematic notation for lower-pair mechanisms based on matrices.," Trans. ASME. J. Appl. Mech., vol. 22, 1955.

[16] D. Schinstock, T. Faddis, and R. Greenway, "Robust Inverse Kinematics Using Damped Least Squares With Dynamic Weighting," ... Intell. Robot. Field, Factory, ..., pp. 861-869, Mar. 1994.

[17] N. Pellegrini et al., "Optimization of power wheelchair control for patients with severe Duchenne muscular dystrophy," Neuromuscul. Disord., vol. 14, no. 5, pp. 297-300, May 2004.

[18] M. Mazo et al., "Electronic control of a wheelchair guided by voice commands," Control Eng. Pract., vol. 3, no. 5, pp. 665674, May 1995.

[19] G. Pačnik, K. Benkič, and B. Brečko, "Voice operated intelligent wheelchair - VOIC," in IEEE International Symposium on Industrial Electronics, 2005, vol. III, pp. 1221-1226.

[20] J. A. Martínez, A. Úbeda, E. Iáñez, J. M. Azorín, and C. PerezVidal, "Multimodal system based on electrooculography and voice recognition to control a robot arm," Int. J. Adv. Robot. Syst., vol. 10, no. 7, p. 283, Jul. 2013.

[21] “Google Cloud Speech-to-text API.” [Online]. Available: https://cloud.google.com/speech-to-text/.

[22] E. D. Guestrin and M. Eizenman, "General theory of remote gaze estimation using the pupil center and corneal reflections," IEEE Trans. Biomed. Eng., vol. 53, no. 6, pp. 1124-1133, 2006.

[23] J. Brooke, "SUS-A quick and dirty usability scale," in Usability evaluation in industry, 1996, pp. 189-194.

[24] A. Mayhew et al., "Development of the Performance of the Upper Limb module for Duchenne muscular dystrophy," Dev. Med. Child Neurol., vol. 55, no. 11, pp. 1038-1045, Nov. 2013.

[25] J. Brooke, "SUS: a retrospective," J. Usability Stud., vol. 8, no. 2, pp. 29-40, 2013.

[26] A. Bangor, P. T. Kortum, and J. T. Miller, "An empirical evaluation of the system usability scale," Int. J. Hum. Comput. Interact., vol. 24, no. 6, pp. 574-594, Jul. 2008.

[27] J. Sauro, "Measuring Usability With The System Usability Scale (SUS)," Meas. Usability, pp. 1-5, 2011. 